\title{
Anisotropy of ionospheric irregularities determined from the amplitude of satellite signals at a single receiver
}

\author{
E. D. Tereshchenko ${ }^{1}$, B. Z. Khudukon ${ }^{1}$, M. O. Kozlova ${ }^{1}$, and T. Nygrén ${ }^{2}$ \\ ${ }^{1}$ Polar Geophysical Institute, 15 Khalturina, 183010 Murmansk, Russia \\ ${ }^{2}$ Department of Physical Sciences, University of Oulu, FIN-90570 Oulu, Finland
}

Received: 1 April 1998 / Revised: 19 August 1998 / Accepted: 26 August 1998

\begin{abstract}
A new method of determining the anisotropy parameters of small-scale irregularities in the ionospheric $\mathrm{F}$ region is presented and experimental results are shown. The method is based on observations of amplitude fluctuations of radio waves transmitted by satellites flying above the $\mathrm{F}$ region. In practice, Russian navigational satellites are used and both the amplitude and the phase of the received signal is measured on the ground level. The method determines both the field-aligned anisotropy and the field-perpendicular anisotropy and orientation of the spatial spectrum of the irregularities, assuming that the contours of constant power have an elliptic shape. A possibility of applying the method to amplitude tomography is also discussed. Using a chain of receivers on the ground level, one could locate the regions of small-scale irregularities as well as determine their relative intensities. Then the large-scale background structures could be mapped simultaneously by means of ordinary ray tomography using the phase observations, and therefore the relations of small-scale and large-scale structures could be investigated.
\end{abstract}

Key words. Ionosphere (auroral ionosphere; ionospheric irregularities; instruments and techniques)

\section{Introduction}

The relations of small-scale irregularities (with scale sizes from about $10 \mathrm{~km}$ down to the ion gyroradius) and inhomogeneities of larger scale play an important role in the physics of the high-latitude ionospheric $\mathrm{F}$ region (for a review, see Tsunoda, 1988). The small-scale structures are generated by plasma instabilities driven by strong

Correspondence to: T. Nygrén ionospheric electric fields and plasma density gradients (for a review, see Keskinen and Ossakow, 1983).

The two main methods of investigating the small-scale ionospheric irregularities at high latitudes are based on coherent scatter and scintillation. In the former case, backscatter from field-aligned irregularities is observed by means of coherent radars with their antennas pointing in such a way that the wave vector of the probing wave will meet the irregularities in a direction perpendicular to the geomagnetic field. The main information given by coherent scatter radars is the intensity and Doppler shift of the scattered signal, which can be used to determine the intensity and line-of-sight phase velocity of the irregularities. By probing a given region separately with two widely spaced radars, the two-dimensional drift velocities can be obtained. The conditions for the generation of the irregularities can be studied by simultaneous incoherent scatter measurements of the ionospheric electric field. At VHF or UHF frequencies this is only possible for E region irregularities (e.g. Nielsen and Schlegel, 1983; Haldoupis et al., 1990; Eglitis et al., 1996) but at $\mathrm{HF}$ frequencies also for $\mathrm{F}$ region irregularities (Baker et al., 1983; Villain et al., 1985).

In the scintillation method the diffraction pattern caused by the ionospheric irregularities is observed on the ground level (for a review on the scintillation of radio waves, see Yeh and Liu, 1982). In this case the probing signal originates from a satellite or a radio star and passes through the whole ionosphere thus containing information on the irregularities from the whole of its path. The changes of the diffraction pattern, which are observed both in the amplitude and in the phase of the signal, are due to the motion of the radio source as well as due to the temporal changes and the motion of the irregularities. A conventional way of studying scintillation is to investigate the two-dimensional correlation function of the diffraction pattern on the ground level, which gives both the drift velocity and the anisotropy of the diffraction pattern. This implies the use of at least three receivers which are separated by suitable distances, typically by several hundred metres. 
In this paper a method based on amplitude fluctuation measurements is presented, which allows the determination of the anisotropy parameters using a single receiver. The field-aligned irregularities at high latitudes cause more intensive amplitude fluctuations when the ray direction lies close to the direction of the geomagnetic field (so called aspect effect). Therefore, the level of the amplitude fluctuations in this direction is expected to be sensitive to the changes of the irregularity parameters. This paper shows the validity of this assumption by comparing the experimental data to theoretical models. It also presents the possibility of determining such parameters as the anisotropy of the high-latitude irregularities and the direction of the anisotropy in the plane perpendicular to the magnetic field.

When several receivers measuring the total electron content are arranged in a chain, the electron density distribution in the vertical plane above the receivers can be determined from the phase observations using tomographic methods. In this paper it will be shown that, with some simplifying assumptions, tomographic methods can, at least in principle, be applied to the amplitude data in order to determine the location and perhaps also the relative intensities of the ionospheric small-scale irregularities. Since phase and amplitude are registered by the same instrument, the background ionisation and the small-scale irregularities could then be determined simultaneously. This would give a new dimension in studying the generation of the irregularities: The location of irregularities could be found by means of amplitude tomography, the background electron density and its gradients would be obtained from ordinary phase tomography and, finally, the electric field could be given by an incoherent scatter radar.

\section{Theory}

We assume that the electron density in the ionospheric plasma is a random continuous function of space. It can be represented in the form

$N(\mathbf{r}, t)=N_{0}(\mathbf{r})+\delta N(\mathbf{r}, t)$,

where $N_{0}(\mathbf{r})$ is the average density and $\delta N(\mathbf{r}, t)$ is the density fluctuation with zero mean. Here the average density is assumed to vary so slowly in space that it would cause no scintillation alone, and the electron density fluctuation is the source of scintillation. The propagation of radio waves in such a medium has been studied in numerous works (e.g. Ishimaru, 1978; Kunitsyn and Tereshchenko, 1992).

We consider a satellite transmitting a radio signal through the underlying ionosphere to a receiver on the ground level. If the density fluctuations $\delta N(\mathbf{r}, t)$ are not negligible, scintillation will be observed both in the phase and in the amplitude of the received signal. In the below theory we will neglect the phase fluctuations and will investigate the amplitude variations only.

At each point in space, the density fluctuation is a stochastic process with some specific frequency spectrum. The fluctuations at different points are not independent, but they correlate. This correlation is expressed by the three-dimensional spatial correlation function

$R_{(3)}(\mathbf{r}, \varrho)=\langle\delta N(\mathbf{r}) \delta N(\mathbf{r}+\varrho)\rangle$.

Its Fourier transform

$\Phi(\mathbf{r}, \boldsymbol{\kappa})=\iint_{-\infty}^{\infty} \int_{(3)}(\mathbf{r}, \varrho) \exp (-i \boldsymbol{\kappa} \cdot \varrho) \mathrm{d}^{3} \rho$

is the three-dimensional spatial spectrum of the fluctuations. Here $\varrho$ is the spatial increment vector, $\boldsymbol{\kappa}$ is the three-dimensional wave number vector and $\Phi(\mathbf{r}, \boldsymbol{\kappa})$ indicates the power spectral density of the fluctuations at the wave number $\boldsymbol{\kappa}$ in the point $\mathbf{r}$.

In the continuation, it is convenient to choose a Cartesian coordinate system with its $z$ axis pointing from the receiver to the satellite and the geomagnetic field vector at the altitudes of the scattering layer to lie in the $y z$ plane. This is shown in Fig. 1. Thus the $x y$ plane will be perpendicular to the radio beam and the $x$ axis will be perpendicular to the geomagnetic field. The angle between the $z$ axis and the direction of the geomagnetic field, denoted by $\Theta$, is obviously a function of $z$. When the satellite travels along its path, the coordinate system will turn accordingly with the satellite motion. At a certain satellite position, the value of $\Theta$ at a fixed height $h$ obtains its minimum value, which is denoted by $\Theta_{\min }$.

The signal observed at the receiver is affected by scattering due to irregularities at or close to the $z$ axis. The signal is statistically controlled by two-dimensional autocorrelation functions of density fluctuations in planes perpendicular to the $z$ direction. These functions are defined by

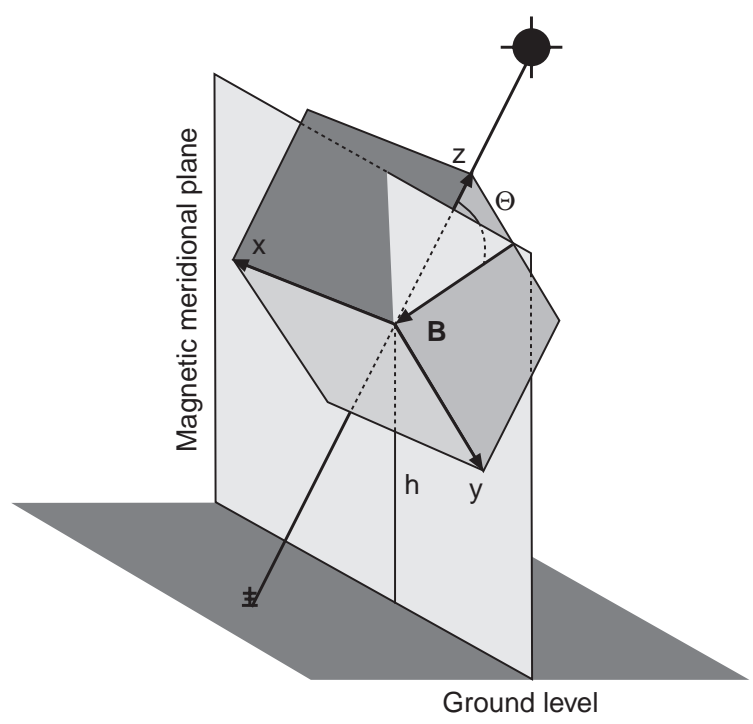

Fig. 1. The geometry and coordinate system for scintillation observations 


$$
\begin{aligned}
R_{(2)}\left(\mathbf{r}, \varrho_{x y}\right) & =\int_{-\infty}^{\infty} R_{(3)}(\mathbf{r}, \varrho) \mathrm{d} \rho_{z} \\
& =\int_{-\infty}^{\infty}\langle\delta N(\mathbf{r}) \delta N(\mathbf{r}+\varrho)\rangle \mathrm{d} \rho_{z},
\end{aligned}
$$

where $\varrho_{x y}=\rho_{x} \mathbf{e}_{x}+\rho_{y} \mathbf{e}_{y}$ and $\rho_{z}$ are the spatial increments perpendicular and parallel to the $z$ axis, respectively. It can be easily shown that

$R_{(2)}\left(\mathbf{r}, \varrho_{x y}\right)=\frac{1}{(2 \pi)^{2}} \iint_{-\infty}^{\infty} \Phi\left(\mathbf{r}, \boldsymbol{\kappa}_{x y}\right) \exp \left(i \boldsymbol{\kappa}_{x y} \cdot \varrho\right) \mathrm{d} \kappa_{x} \mathrm{~d} \kappa_{y}$,

where $\boldsymbol{\kappa}_{x y}=\kappa_{x} \mathbf{e}_{x}+\kappa_{y} \mathbf{e}_{y}$ is the fluctuation wave number vector perpendicular to the $z$ axis. This indicates that $R_{(2)}\left(\mathbf{r}, \varrho_{x y}\right)$ contains information on those density fluctuations which have their wave fronts parallel to the $z$ axis.

Using the Markov approximation (Rytov et al., 1978), the logarithmic mean relative signal intensity at the receiver is

$X=\ln \left\langle\frac{A^{2}}{A_{0}^{2}}\right\rangle=-\frac{\lambda^{2} r_{e}^{2}}{2} \int_{z_{l}}^{z_{u}} R_{(2)}\left(z \mathbf{e}_{z}, 0\right) \mathrm{d} z$

where $A$ is the observed amplitude, $A_{0}$ is the amplitude as it would be without the irregularities, $\lambda$ is the wave length of the probing signal, $r_{e}$ is the classical electron radius, and $z_{l}$ and $z_{u}$ are the lower and upper limits of the region of irregularities at the $z$ axis. Using Eq. (5) this can be put in the form

$X=-\frac{\lambda^{2} r_{e}^{2}}{8 \pi^{2}} \int_{z_{l}}^{z_{u}} \mathrm{~d} z \iint_{-\infty}^{\infty} \Phi\left(z \mathbf{e}_{z}, \boldsymbol{\kappa}_{x y}\right) \mathrm{d} \kappa_{x} \mathrm{~d} \kappa_{y}$.

Hence the medium behaves like a stack of thin diffracting plates, which lie perpendicular to the line of sight. Some of the incident energy is scattered aside from the $z$ axis in each plate, so that the average intensity is reduced exponentially much like in absorption. No secondary or higher order scattering is taken into account. The scattering is due to those wave fronts of the density fluctuation which lie parallel to the $z$ axis.

If the surfaces of constant power of the spatial spectrum are ellipsoids with one of their principal axes pointing in the direction of the geomagnetic field, the spectrum depends on three anisotropy parameters; the field aligned anisotropy $\alpha$, the perpendicular anisotropy $\beta$ and the orientation angle $\Psi$ of the perpendicular ellipse. Then, if $A_{0}$ and the shape of the spatial spectrum were known, measuring the fluctuations of $A$ could in principle give a possibility of determining the anisotropy parameters by solving the inversion problem posed by Eq. (7). The value of $A_{0}$ is not known, however, since we only measure the fluctuating amplitude. Attempts have been made to estimate $A_{0}$ by means of filtering or loworder polynomial fits, but these methods actually give (weighted) averages of $A$ rather than experimental estimates of $A_{0}$.

The error made in using the mean value of $A$ instead of $A_{0}$ can be evaluated as follows. It is well known that the logarithmic relative amplitude

$\chi=\ln \frac{A}{A_{0}}$

obeys Gaussian distribution in the case of weak scattering (see e.g. Goodman, 1985). If no absorption and scattering in backward direction is assumed, the energy conservation implies that the mean intensity is independent of height. This condition together with the Gaussian distribution of $\chi$ gives a mean value

$\langle\chi\rangle=-\sigma_{\chi}^{2}$,

where $\sigma_{\chi}^{2}$ is the variance of $\chi$. Hence the energy conservation implies that the ratio of the mean value of $\chi$ and its variance is fixed. Using this result, one can easily show that the mean amplitude is

$\langle A\rangle=A_{0} \exp \left(-\frac{\sigma_{\chi}^{2}}{2}\right)$.

Therefore, if $A_{0}$ is replaced by $\langle A\rangle$ in estimating $X$, the measured quantity will actually be

$X_{e}=\ln \frac{\left\langle A^{2}\right\rangle}{\langle A\rangle^{2}}=\ln \left\langle\frac{A^{2}}{A_{0}^{2}}\right\rangle-\ln \frac{\langle A\rangle^{2}}{A_{0}^{2}}=X+\sigma_{\chi}^{2}$

so that the value of $X$ will always be overestimated by $\sigma_{\chi}^{2}$.

In this paper we use a different approach. Instead of $X$ or $X_{e}$, let us consider the quantity

$\chi_{e}=\left\langle\ln \frac{A}{\langle A\rangle}\right\rangle$.

Using Eqs. (8)-(10) it is readily seen that

$\chi_{e}=\left\langle\ln \frac{A}{A_{0}}\right\rangle-\ln \frac{\langle A\rangle}{A_{0}}=\langle\chi\rangle+\frac{\sigma_{\chi}^{2}}{2}=-\frac{\sigma_{\chi}^{2}}{2}$.

The variance of $\chi$ can now be obtained by using the Rytov approximation. This gives

$\chi_{e}=-\frac{\lambda^{2} r_{e}^{2}}{8 \pi^{2}} \int_{z_{l}}^{z_{u}} \mathrm{~d} z \iint_{-\infty}^{\infty} \Phi\left(z \mathbf{e}_{\mathbf{z}}, \boldsymbol{\kappa}_{x y}\right) \sin ^{2} \frac{R_{F}^{2} \kappa_{x y}^{2}}{4 \pi} \mathrm{d} \kappa_{x} \mathrm{~d} \kappa_{y}$,

where $z_{0}$ is the distance between the transmitter and the receiver and $R_{F}=\left[\lambda z\left(z_{0}-z\right) / z_{0}\right]^{1 / 2}$ is the radius of the first Fresnel zone. A comparison with Eq. (7) reveals a close similarity between the two equations. The only difference in the integrals is the factor $\sin ^{2}\left(R_{F}^{2} \kappa_{x y}^{2} / 4 \pi\right)$ in Eq. (14). The quantity $\chi_{e}$ can be determined from the observed amplitude fluctuations and, due to its direct relationship with $\sigma_{\chi}^{2}$, it is a convenient tool for studying the properties of small-scale irregularities.

It is convenient to present the spatial spectrum in the form 
$\Phi(\mathbf{r}, \boldsymbol{\kappa})=\sigma_{N}^{2}(\mathbf{r}) \Phi_{0}(\mathbf{r}, \boldsymbol{\kappa})$,

where $\sigma_{N}^{2}(\mathbf{r})$ is the variance of the density fluctuations and $\Phi_{0}(\mathbf{r}, \boldsymbol{\kappa})$ is the normalised spectral density (i.e. its total power is equal to unity). With this notation Eq. (14) can be written as

$$
\begin{aligned}
\chi_{e}= & -\frac{\lambda^{2} r_{e}^{2}}{8 \pi^{2}} \int_{z_{l}}^{z_{u}} \mathrm{~d} z \sigma_{N}^{2}(z) \\
& \times \iint_{-\infty}^{\infty} \Phi_{0}\left(z \mathbf{e}_{\mathbf{z}}, \boldsymbol{\kappa}_{x y}\right) \sin ^{2} \frac{R_{F}^{2} \kappa_{x y}^{2}}{4 \pi} \mathrm{d} \kappa_{x} \mathrm{~d} \kappa_{y} .
\end{aligned}
$$

Here the double integral in the wave number space depends only on the shape of the fluctuation spectrum but not on the strength of the fluctuations.

The use of this result for our purpose implies an explicit definition of the shape of the spectrum. We shall adopt the widely used power law spectral shape

$$
\begin{aligned}
\Phi_{0(p)}(\mathbf{r}, \boldsymbol{\kappa})= & \frac{\alpha \beta L_{0}^{3} \Gamma(p / 2)}{2 \pi \Gamma(3 / 2) \Gamma[(p-3) / 2]} \\
& \times\left[1+\left(\frac{L_{0}}{2 \pi}\right)^{2}\left(\alpha^{2} \kappa_{\|}^{2}+\beta^{2} \kappa_{x \perp}^{2}+\kappa_{y \perp}^{2}\right)\right]^{-p / 2}
\end{aligned}
$$

In this formula $\Gamma$ is the gamma function, $p$ is the power index, $L_{0}$ is the scale size of the irregularities, and $\kappa_{\|}, \kappa_{x \perp}$ and $\kappa_{y \perp}$ are the components of $\boldsymbol{\kappa}$ in the directions parallel and perpendicular to the geomagnetic field. Assuming $\alpha>1$ and $\beta>1$ the irregularities are elongated in the directions of the geomagnetic field and the $x \perp$ axis. In the case of symmetric irregularities the choice of the perpendicular axes is arbitrary. The anisotropy parameters $\alpha$ and $\beta$ actually indicate the relative scale sizes in the field-aligned and field-perpendicular directions, while the absolute scale size is given by $L_{0}$. The present method will allow the determination of the anisotropy parameters, but the absolute scale size remains undetermined.

For simplicity, we first assume that the spectrum is isotropic around the field direction, i.e. $\beta=1$ (this assumption will be relaxed later). Another simplification is given by the fact that, when VHF frequencies are used in radio probing experiments, the first Fresnel's radius does not exceed $1 \mathrm{~km}$. Since the external scale of the irregularities $L_{0}$ is estimated to be tens of kilometres (see e.g. Aarons, 1982), the ratio $R_{F} / L_{0}$ is small. Then the spectral shape in Eq. (17) can be replaced by

$\Phi_{0(p)}(\mathbf{r}, \boldsymbol{\kappa})=\frac{\Phi_{0(p)}(\mathbf{r}, 0)}{\left[\left(L_{0} / 2 \pi\right)^{2}\left(\kappa_{\perp}^{2}+\alpha^{2} \kappa_{\|}^{2}\right]^{p / 2}\right.}$,

where $\kappa_{\perp}^{2}=\kappa_{x \perp}^{2}+\kappa_{y \perp}^{2}$ and

$\Phi_{0(p)}(\mathbf{r}, 0)=\frac{\alpha L_{0}^{2} \Gamma(p / 2)}{2 \pi \Gamma(3 / 2) \Gamma[(p-3) / 2]}$.

The use of this spectral shape for power indices $0<p<4$ in calculating the integrals in Eq. (16) leads to

$$
\begin{aligned}
\chi_{e(p)}= & -\frac{\lambda^{2} r_{e}^{2} \alpha L_{0}^{3-p} \pi^{(p-1) / 2}}{4 \Gamma[(p-3) / 2] \sin p \pi / 4} \int_{z_{l}}^{z_{u}} \mathrm{~d} z \\
& \times \frac{\sigma_{N}^{2}(z)}{\left[1+\left(\alpha^{2}-1\right) \sin ^{2} \Theta(z)\right]^{1 / 2}} R_{F}^{p-2}(z) \\
& \times F\left[1-p / 2,1 / 2,1, \frac{\left(\alpha^{2}-1\right) \sin ^{2} \Theta(z)}{1+\left(\alpha^{2}-1\right) \sin ^{2} \Theta(z)}\right]
\end{aligned}
$$

where $\mathrm{F}$ is the hypergeometric function (see e.g. Abramowitz and Stegun, 1970 or Gradshteyn and Ryzhik, 1965).

The value of the argument of the hypergeometric function in Eq. (20) is equal to 0 when $\sin \Theta=0$ and approaches 1 when $\sin \Theta \neq 0$ and $\alpha$ approaches infinity. Therefore, since the irregularities are field-aligned, there is a small range of angles $\Theta(z)$ around the local magnetic zenith where the $z$ dependence of the hypergeometric function must be taken into account.

Because $\alpha \gg 1$, for all other directions the hypergeometric function can be replaced by its limiting value

$F(1-p / 2,1 / 2,1,1)=\frac{\Gamma[(p-1) / 2]}{\Gamma(1 / 2) \Gamma(p / 2)}$.

This approximation helps in understanding the importance of the hypergeometric function in Eq. (20). In the practical calculations the true values of the hypergeometric function were used, however.

For a given position of the satellite, the slowly varying function $R_{F}^{p-2}(z)$ can be easily calculated. Furthermore, a geomagnetic field model gives $\sin \Theta$, and therefore the integral in Eq. (20) can be calculated for a model $\sigma_{N}^{2}(z)$ and a choice of the parameter $\alpha$. Then, in principle, it will be possible to reconstruct $\chi_{e}$ by varying $\alpha$ and finding the value which gives the closest agreement with the observational results.

One may suspect that this result is sensitive to the choice of the spectrum model. Some idea of the effect of the spectral shape is obtained, if the power law is replaced by the Gaussian spectrum

$\Phi_{0(G)}(\mathbf{r}, \boldsymbol{\kappa})=\frac{\alpha L_{0}^{3}}{\pi^{3 / 2}} \exp \left[-\left(\frac{L_{0}}{2 \pi}\right)^{2}\left(\kappa_{\perp}^{2}+\alpha^{2} \kappa_{\|}^{2}\right)\right]$.

When this function is inserted in Eq. (16), the integration gives, with an accuracy of the order of $\left(R_{F} / L_{0}\right)^{2}$,

$$
\begin{aligned}
\chi_{e(G)}= & -\pi^{3 / 2} r_{e}^{2} \lambda^{2} \alpha L_{0} \\
& \times \int_{z_{l}}^{z_{u}} \mathrm{~d} z \frac{\sigma_{N}^{2}(z)}{\left[1+\left(\alpha^{2}-1\right) \sin ^{2} \Theta(z)\right]^{1 / 2}} \\
& \times\left(\frac{R_{F}}{L_{0}}\right)^{4} F\left[-2, \frac{1}{2}, 1, \frac{\left(\alpha^{2}-1\right) \sin ^{2} \Theta(z)}{1+\left(\alpha^{2}-1\right) \sin ^{2} \Theta(z)}\right]
\end{aligned}
$$

A comparison of $\chi_{e(p)}$ and $\chi_{e(G)}$ in Eqs. (20) and (23) indicates that the integrands are identical for $p=6$ so that, excluding the scaling factor, the results are the same. Hence $\chi_{e(p)}$ and $\chi_{e(G)}$ behave essentially in a 
similar way, i.e. $\chi_{e}$ does not depend strongly on the shape of the fluctuation spectrum.

It is interesting to notice that the spectral shapes in Eqs. (18) and (22) give expressions to the double integral in Eq. (7) which are otherwise similar to those in Eqs. (20) and (23), but without the hypergeometric functions and the factors containing the Fresnel's radius.

Since the irregularities are not symmetric around the direction of the geomagnetic field, the simplified theory with $\beta=1$ is not general enough for real data analysis. The above derivations can be repeated for a general case also. The results are quite complicated and therefore $\chi_{e}$ is given here only for the spectrum obeying the power law. When the irregularities are anisotropic in a plane perpendicular to the field direction, we then have

$$
\begin{aligned}
\chi_{e(p)}= & -\frac{\lambda^{2} r_{e}^{2} \alpha \beta L_{0}^{3-p} \pi^{(p-1) / 2}}{2 \Gamma[(p-3) / 2] \sin p \pi / 4} \int_{z_{l}}^{z_{u}} \mathrm{~d} z \\
& \times \frac{\sigma_{N}^{2}(z)}{\sqrt{1+\gamma^{2}(z)}} R_{F}^{p-2}(z) F\left[1-\frac{p}{2}, \frac{1}{2}, 1, \frac{\gamma(z)}{1+\gamma(z)}\right] \\
& \times\left[C(z)-\sqrt{A^{2}(z)+B^{2}(z)}\right]^{-p / 2},
\end{aligned}
$$

where

$$
\gamma=\frac{2 \sqrt{A^{2}(z)+B^{2}(z)}}{C(z)-\sqrt{A^{2}(z)+B^{2}(z)}}
$$

and

$$
\begin{aligned}
A= & \frac{1}{2}\left[\left(\alpha^{2}-1\right) \sin ^{2} \Theta(z)\right. \\
& \left.+\left(\beta^{2}-1\right)\left(\sin ^{2} \Psi \cos ^{2} \Theta(z)-\cos ^{2} \Psi\right)\right] \\
B= & \left(\beta^{2}-1\right) \sin \Psi \cos \Psi \cos \Theta(z) \\
C= & 1+\frac{1}{2}\left[\left(\alpha^{2}-1\right) \sin ^{2} \Theta(z)\right. \\
& \left.+\left(\beta^{2}-1\right)\left(\sin ^{2} \Psi \cos ^{2} \Theta(z)+\cos ^{2} \Psi\right)\right] .
\end{aligned}
$$

Here $\Psi$ is the angle of orientation of the irregularities in the plane perpendicular to the geomagnetic field, $\Psi=0$ and $\Psi=180^{\circ}$ indicating geomagnetic westward and eastward directions, respectively. The above theory can be applied in determining the anisotropy parameters of the ionospheric irregularities from observed amplitude fluctuations. Experimental estimates for $\chi_{e}$ will be first calculated from observed amplitude fluctuations, and the resulting time series will be then fitted to theoretical values of $\chi_{e}$. Since the absolute scale size $L_{0}$ appears in Eqs. (20) and (24) only in the product $L_{0}^{3-p} \sigma_{N}^{2}$, it is inseparable from the absolute value of the fluctuation variance, and therefore it cannot be determined. A noticeable feature of this method is that a single receiver is sufficient in determining the anisotropy parameters.

\section{Experimental setup}

The above theory was applied in analysing data collected in the Russian-Finnish tomographic campaign in November 1995. The experimental setup consisted of
Russian navigational satellites and a chain of four satellite receivers extending from the north of Norway to the south of Finland. A more detailed description of the campaign is given by Nygrén et al., (1996).

The experimental setup was originally constructed for measuring the total electron content by means of the conventional difference Doppler method with coherent waves at two frequencies (150 and $400 \mathrm{MHz})$. A brief description of the receiving system is given by Chernyakov et al., (1993) and Markkanen et al., (1995). For the present campaign the receivers were developed to allow the determination of the signal amplitude at $150 \mathrm{MHz}$ frequency for the purpose of scintillation research.

An extra amplitude channel was added to a direct measurement of the object wave so that both phase and amplitude can be registered. Data from the amplitude channel can be compared with the amplitude given by the quadrature detector. Phase observations from this same data base were used in previous studies on satellite radiotomography. Unlike those works, the present one only makes use of the amplitude data. The data selected for analysis come from the two northernmost sites in the chain, i.e. Tromsø $\left(69.662^{\circ} \mathrm{N}, 18.940^{\circ} \mathrm{E}\right)$ and Esrange $\left(67.877^{\circ} \mathrm{N}, 21.064^{\circ} \mathrm{E}\right)$.

The satellites used in the experiment fly at an altitude of about $1000 \mathrm{~km}$ approximately along a geomagnetic meridian during their southward passages. Of course, they only rarely fly precisely above the receiver chain, and therefore the situation is usually like that shown in Fig. 1. This means that the satellite only seldom passes the local magnetic zenith, and therefore $\Theta_{\min }$, the smallest angle between the satellite direction and the magnetic field direction at the altitude of the scattering layer, usually clearly departs from zero.

\section{Data analysis}

In those cases when the satellite trajectory lies nearly in the geomagnetic meridional plane of the receiver, $\Theta_{\min } \approx 0$ while the satellite passes the local magnetic zenith. Then scintillation due to weak irregularities can only be observed close to this direction, and the data are affected merely by the field-aligned anisotropy. Thus the field-perpendicular anisotropy cannot be determined but the field-aligned anisotropy can still be obtained by means of Eq. (20) [actually the same result is given by Eq. (24) with a fixed value $\beta=1]$. If the satellite trajectory departs form the geomagnetic meridional plane of the receiver, $\Theta_{\min }$ is never zero. In this case the data are affected not only by the field-aligned but also by the field-perpendicular anisotropy. Then enhanced scintillation is observed at directions close to $\Theta_{\min }$, and both $\alpha$ and $\beta$ can be determined using Eq. (24).

The data were sampled at a frequency of $50 \mathrm{~Hz}$. The fact that the sampling interval is close to the correlation times of metre-scale irregularities poses no problem, since the contribution to amplitude scintillation comes mainly from the more slowly varying irregularities with scale sizes of tens or hundreds of metres, i.e. from scales longer than the 3-m wave length of the probing wave. 

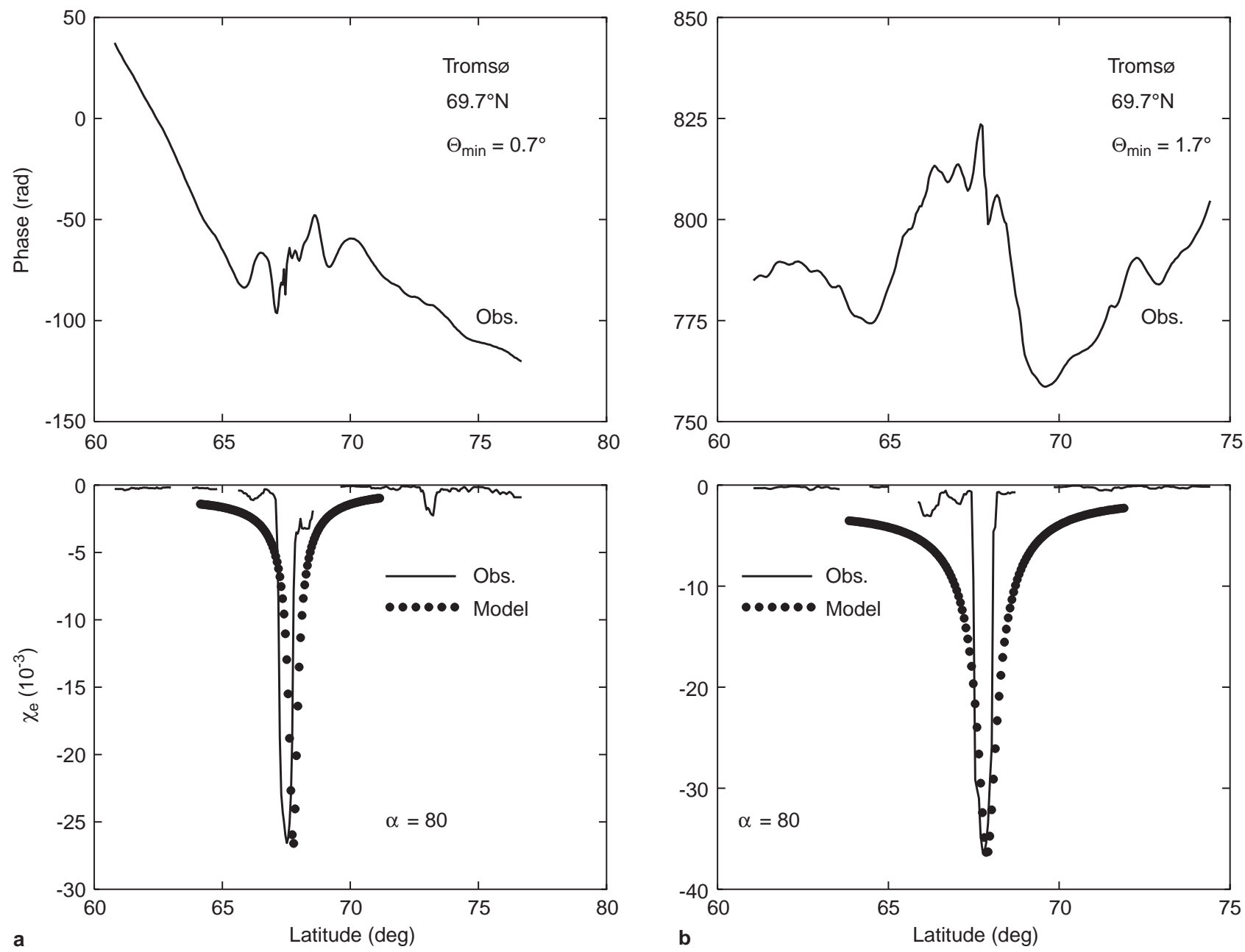

Fig. 2. Examples for phase (top panels) and $\chi_{e}$ (bottom panels) from two satellite passages at Troms (continuous lines) together with results of theoretical fits to the amplitude data (dots). The horizontal axis indicates the geographical latitude of the satellite. In these cases the satellite flies close to the geomagnetic meridional plane of the receiver

( $\Theta_{\min }$ is small), and therefore the spectrum is assumed to be symmetric around the geomagnetic field direction in theoretical fits. The assumed spectral shape is a power law with a power index $11 / 3$, The best values of $\alpha$ are indicated in the bottom panels

The amplitude data were first processed to obtain $\chi_{e}$. For this purpose the time series were divided into intervals of 500 data points, i.e. intervals of $10 \mathrm{~s}$ in the time domain at the applied $50 \mathrm{~Hz}$ sampling frequency. A linear fit was then made to each interval and, for each sampling time, the fitted value $\langle A\rangle_{e}$ was taken as the experimental estimate of the mean value $\langle A\rangle$. Next the quantity $\ln \left(A /\langle A\rangle_{e}\right)$ was determined for each sample in the interval and, finally, their averages $\left\langle\ln \left(A /\langle A\rangle_{e}\right)\right\rangle$ were calculated for each interval. These values are used as experimental estimates of $\chi_{e}$. This procedure gives $\chi_{e}$ at a resolution of $10 \mathrm{~s}$. Since the antenna pattern affects the observed amplitudes, it was carefully checked that the pattern was smoothly behaving within the scale of irregularities. This guarantees that the observed changes come from the irregularities rather than from rapid variations of the antenna pattern.

In order to choose the spectral model to be used in the analysis, power spectra of the original data were calculated and the results were compared with theoretical power law spectra. It turned out that in all cases the

power index varied between 3 and 4 . Within this range of index values the spectral shape depends only weakly on the power index and therefore a single value $p=11 / 3$, which is commonly used in literature, was adopted for simplicity.

Once the power index is selected, $\chi_{e(11 / 3)}$ can be computed for various satellite directions using Eq. (24) or Eq. (20). Then it is possible to compare the observed behaviour of $\chi_{e}$ with theoretical values $\chi_{e(11 / 3)}$ calculated for different values of $\alpha, \beta$ and the orientation angle $\Psi$. This implies a choice of a model for the variance $\sigma_{N}^{2}(z)$. A constant variance within the altitude range 250-350$\mathrm{km}$ altitudes was used in the analysis. Testing different models indicates that the result depends only slightly on the height and thickness of the layer within the horizontally limited region where the effect of spherical geometry can be neglected. The value of the variance was adjusted to give the same depths of the minima in $\chi_{e(11 / 3)}$ and the experimental curve $\chi_{e}$.

The procedure of fitting was briefly as follows. Since it is known that $\alpha$ can vary roughly from 10 to 100 , 
several tens of values of $\alpha$ were chosen from this range. Then $\beta$ was varied from 2 to 30 or 40 for each value of $\alpha$ and keeping the orientation of the perpendicular anisotropy in east-west direction. Next, those values of $\alpha$ and $\beta$ which give the closest fit were used in calculations where the angle of orientation $\Psi$ was varied by $\pm 40^{\circ}$ from the east-west direction. The angle giving the best fit was then used in finding new best-fit values of $\alpha$ and $\beta$, which were again used to find a new value of $\Psi$, etc. The iteration was continued until the results no more changed.

\section{Results}

Results from four cases where the satellite nearly passes the local magnetic zenith are shown in Figs. 2 and 3. The first three of these examples are from Tromsø, which is the northernmost site in the receiver chain, and the last one from a more southern site at Esrange. The continuous lines in the upper and lower panels contain the observed phases and values of $\chi_{e}$ as functions of the geographical latitude of the satellite. The best fits of $\chi_{e(11 / 3)}$ are shown by dotted curves.
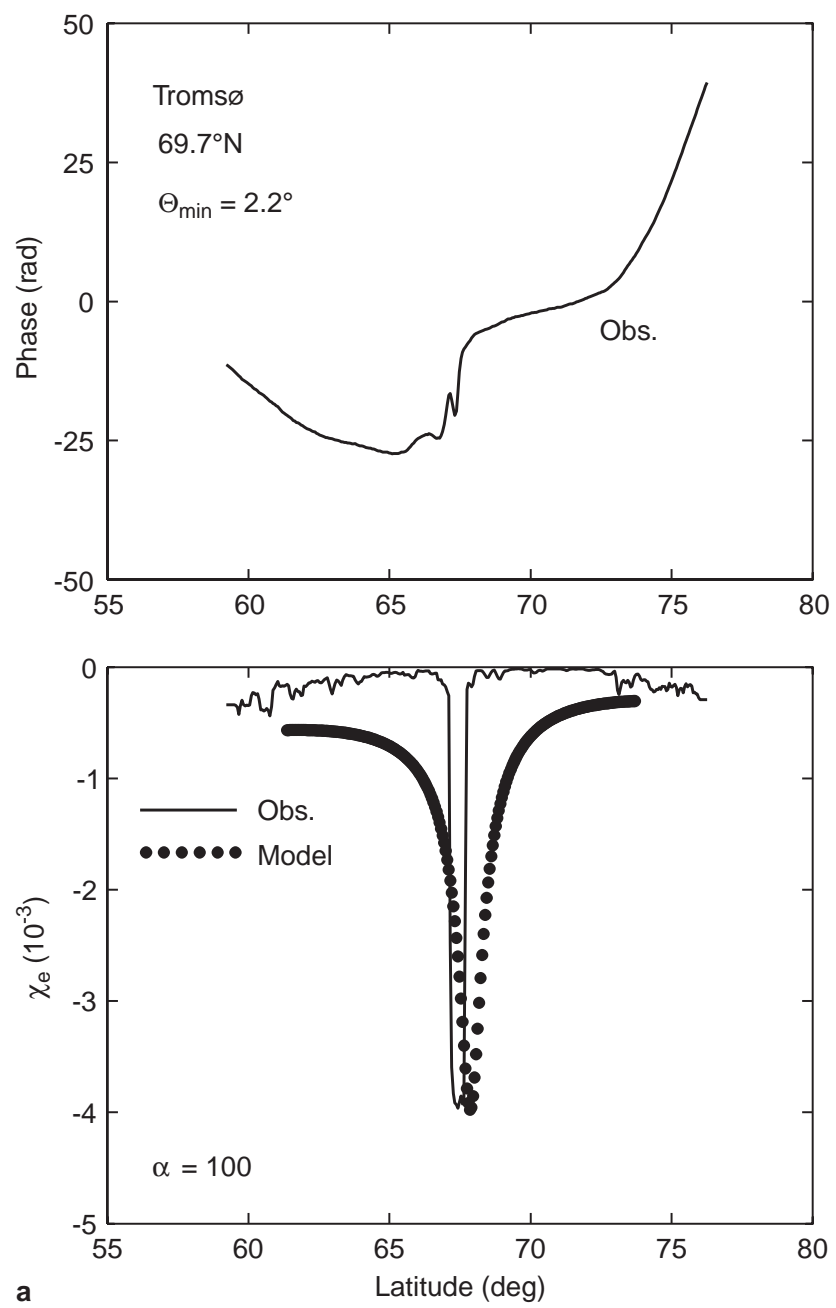

In these figures the scintillation is always observed within a short latitudinal range of satellite positions corresponding to a narrow cone around the geomagnetic field direction. The phase curves show rapid changes at the same latitudes indicating the presence of strong horizontal electron density gradients.

Since $\Theta_{\min }$ is small in these cases, $\chi_{e}$ is much more sensitive to the field-aligned anisotropy than to the perpendicular anisotropy. This means that the assumption $\beta=1$ must be made in the analysis, and only $\alpha$ can be determined. In Fig. 2 the best fit gives $\alpha=80$ for the parallel anisotropy parameter in both cases, whereas in Fig. 3 the anisotropy at Esrange is weaker $(\alpha=35)$ than in Tromsø $(\alpha=100)$.

When comparing the positions and widths of the theoretical and experimental peaks, the best agreement is met in Fig. 3b, where $\Theta_{\min }=0.3^{\circ}$. An analysis of a more extensive data set shows that a good agreement is always obtained, if $\Theta_{\min }<0.5^{\circ}$. If $\Theta_{\min }$ is greater, disagreement will be observed either in the position or the width of the peak. This is clearly seen in Figs. $2 b$ and 3a with $\Theta_{\min }=1.7^{\circ}$ and $\Theta_{\min }=2.2^{\circ}$, respectively. Even in Fig. 2a, where $\Theta_{\min }=0.7^{\circ}$, some disagreement in the peak position is observed.
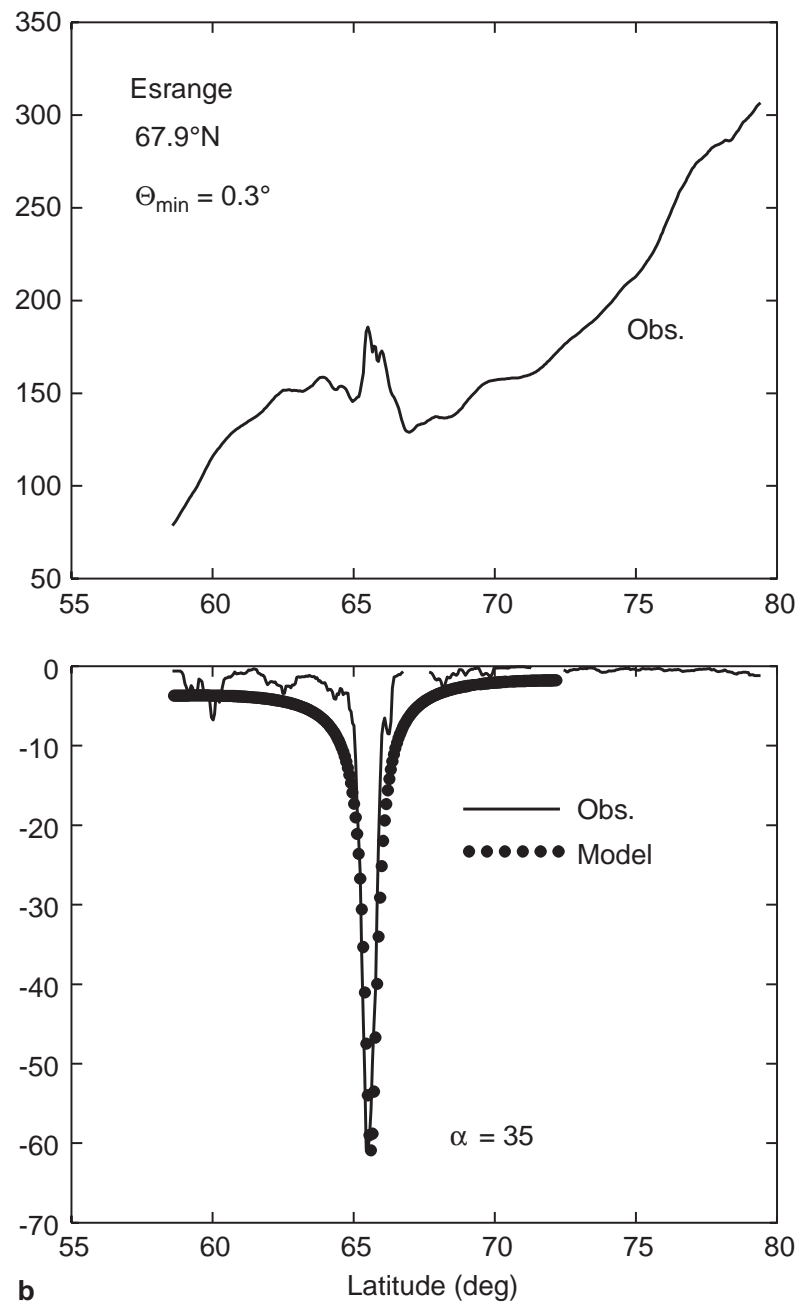

Fig. 3. Same as Fig. 2 for a satellite passage at Tromsø and the other at Esrange 

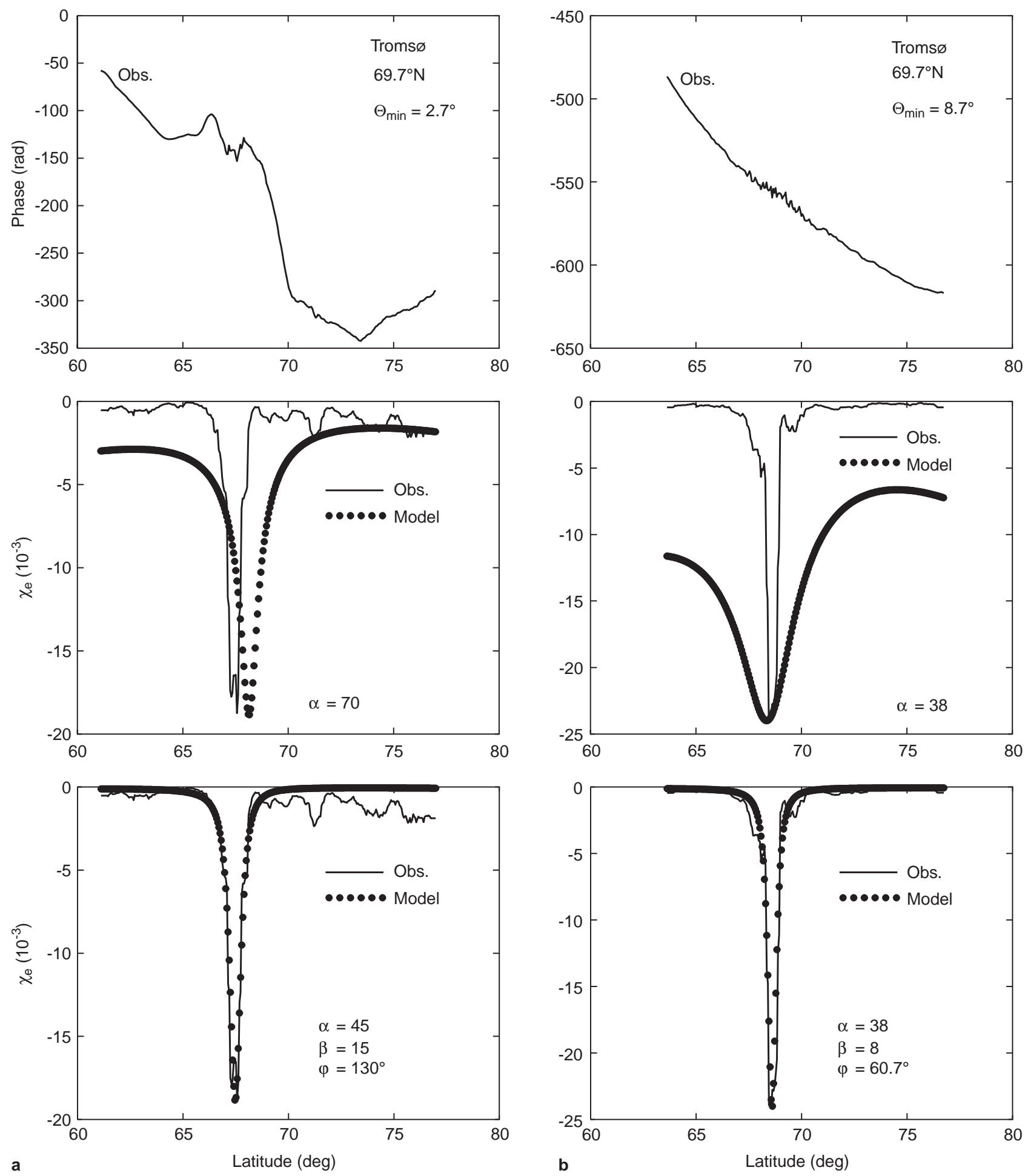

Fig. 4. Examples for phase (top panels) and $\chi_{e}$ (middle and bottom panels) from two satellite passages at Tromsø (continuous lines) together with results of theoretical fits to the amplitude data (dots). The fits in the middle and bottom panels are obtained using spectra which are symmetric and asymmetric around the geomagnetic field, respectively. In each case the spectral shape obeys power law with a

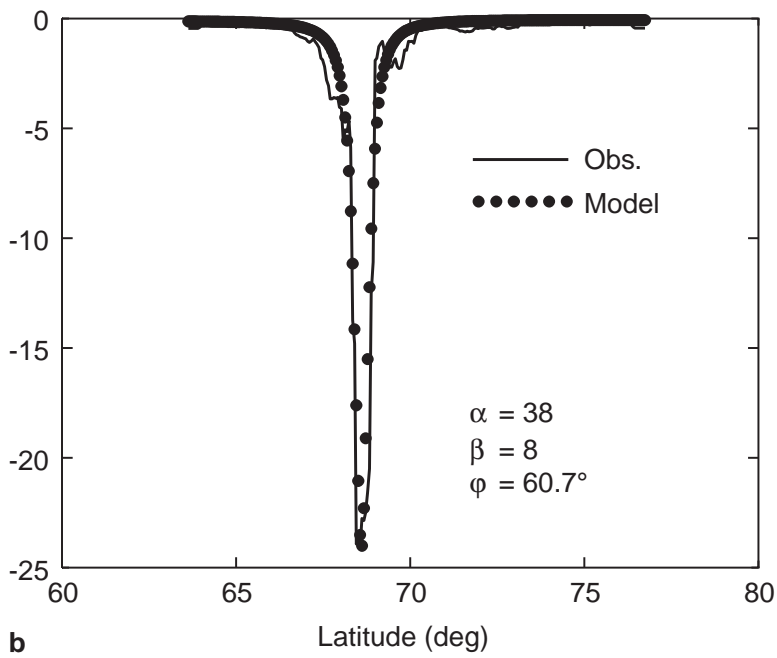

power index 11/3. The best values of $\alpha$ and $\beta$ are indicated in respective panels. In the bottom panels the orientation of the perpendicular anisotropy is given by $\varphi$, which is the directional angle of the horizontal projection of the axis of perpendicular anisotropy, measured eastwards starting from geographic North

A second set of examples, with greater values of $\Theta_{\min }$, is portrayed in Figs. 4 and 5. Just like in the above cases, strong peaks of scintillation are observed within a small range of satellite positions. The enhanced values outside

the main peaks are either due to noise or such strong inhomogeneities that they are capable of producing scintillation in all directions. The phase curves indicate that in some cases the scintillation is associated with 

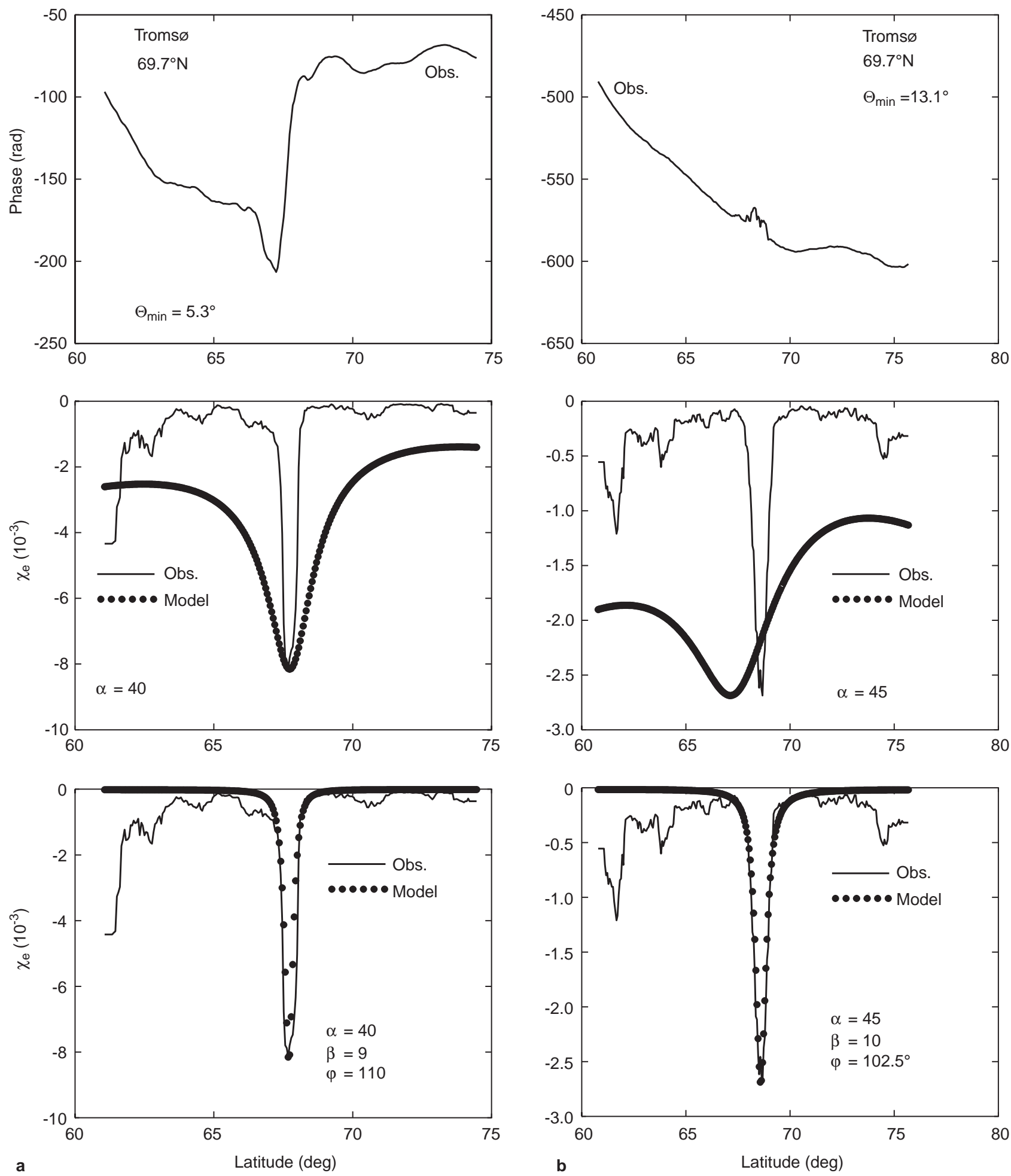

Fig. 5. Same as Fig. 4

large horizontal electron density gradients (Figs. 4a and 5a) but in other cases, especially in Fig. 4b, the irregularities are superimposed on a smoothly behaving phase curve. Unlike in Figs. 2 and 3, the peaks are not always met at the same latitude. This results from a geometrical effect due to the case-to-case variation of $\Theta_{\min }$ and the orientation of perpendicular anisotropy.
The centre panels in Figs. 4 and 5 show theoretical best fits calculated using Eq. (20), i.e. assuming perpendicular isotropy. In all cases the results are essentially worse than those in Figs. 2 and 3. The closest agreement is met in Fig. 4a, where $\Theta_{\min }$ is smallest. The disagreement is a clear indication of the effect of perpendicular anisotropy on scintillation observed on the ground level. 
The results of theoretical fits obtained using Eq. (24), i.e. by taking into account the perpendicular anisotropy, are plotted in the bottom panels of Figs. 4 and 5 . Regardless of the value of $\Theta_{\min }$, an excellent agreement is now observed in all cases. The agreement is even better than in the previous best case in Fig. $3 \mathrm{~b}$ in the sense that the peak remains thin all the way up close to the zero level.

In all cases shown in Figs. 4 and 5, the field-aligned anisotropy parameter is of the order of $40-45$ and the perpendicular parameter of the order of 10-15. Instead of $\Psi$, the direction of the perpendicular anisotropy is expressed in terms of the directional angle of the horizontal projection of the axis of perpendicular anisotropy. This is measured eastward from geographic North and it also shows considerable variation from case to case.

Finally in Fig. 6, three examples from Esrange are presented. Here the dip angle is smaller than in Tromsø. The examples are chosen to cover a wide range of the values of $\Theta_{\min }$. Also here a good agreement by observations and theoretical fits is found. The values of $\alpha$ and $\beta$ are of the same order as in Figs. 4 and 5, and variation of the directional angle is observed also here.

\section{Discussion}

A new possibility of determining the parameters of anisotropy of ionospheric irregularities is demonstrated in this paper. A central point in this method is that a single receiver is sufficient in providing both the fieldaligned anisotropy and field-perpendicular anisotropy as well as its orientation. The method can be applied when inhomogeneities are weak and they are present in the region where the proper geometrical conditions are valid for the probing wave. The latter means that the largest density gradients of the inhomogeneities must be approximately perpendicular to the ray from the satellite to the receiver. Then the probing wave passes through a thick grating created by the inhomogeneities, and amplitude scintillation is observed only within a narrow range of scattering angles. Therefore fitting to a theoretical model can be made assuming a constant variance of the density fluctuations.

A more conventional way of investigating the anisotropy of ionospheric fluctuations is studying the statistical properties of the diffraction pattern of the probing wave on the ground level (see e.g. Briggs and Maude, 1978; Khudukon et al., 1994 and Woodman, 1995). This method, however, implies at least three closely-spaced receivers. Furthermore, it only actually provides the anisotropy of the diffraction pattern, not the field-aligned and perpendicular anisotropies of the irregularities. A benefit of the multireceiver method is that it is capable of giving the drift speed of the diffraction pattern, which cannot be done using a single receiver. Also, it is not limited to the existence of weak irregularities precisely at a proper location, but it is applicable for other directions as well, provided the irregularities are strong enough to cause sufficient scintillation. One should notice that the present method
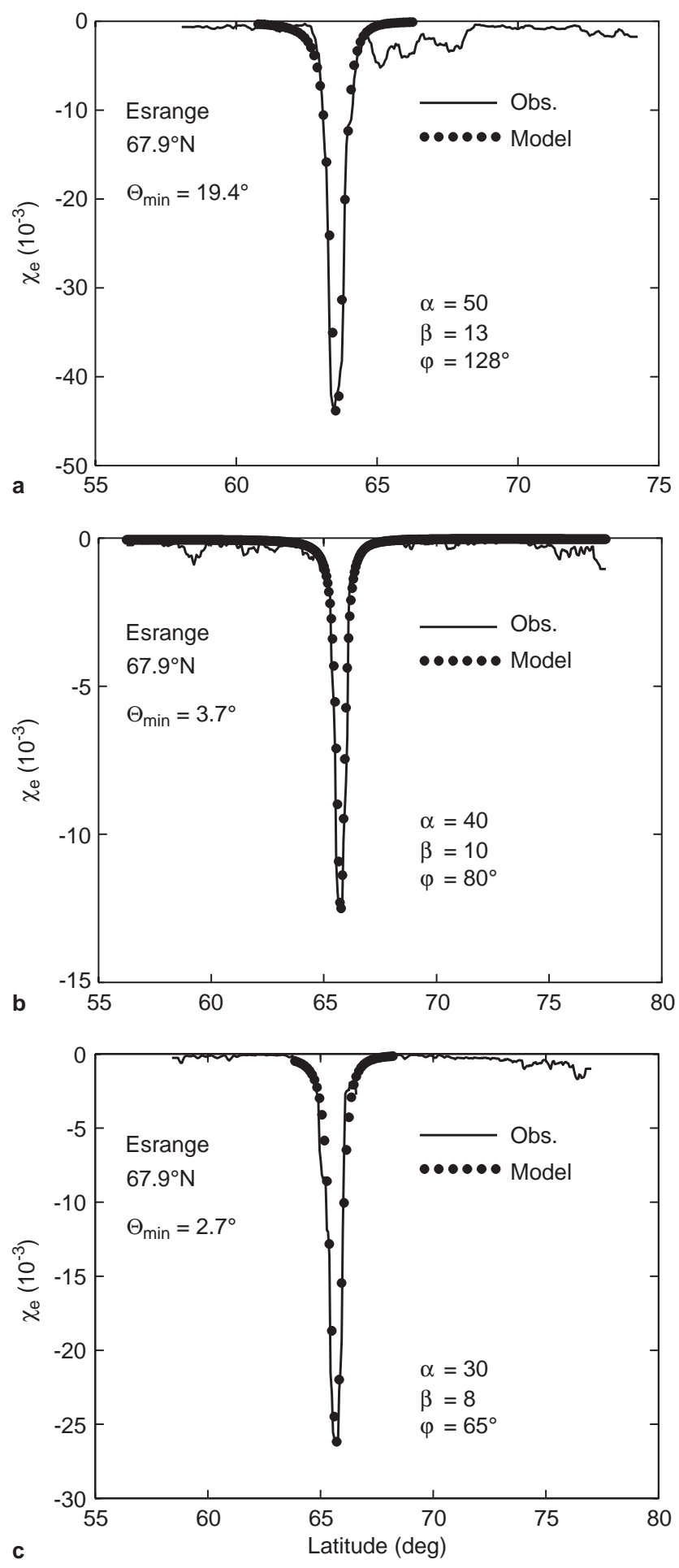

Fig. 6. Examples of amplitude observations and fitted results from three satellite passages at Esrange. The fitting is similar to that in the bottom panels of Figs. 4 and 5

is well applicable in multireceiver experiments as well. Hence, combining the two analysis method could give a more complete picture of the plasma irregularities causing scintillation.

An unusual feature in the present method is that it is best applicable when the satellites do not pass the receiver close to the local zenith. If this happens, the 
scintillation is overwhelmingly controlled by the stronger field-aligned anisotropy so that the field-perpendicular anisotropy cannot be determined properly. The above examples also indicate that in such cases the theoretical fits are not so good as in cases where the satellite has passed the receiver at greater distances.

Although derived for the purpose of determining the anisotropy, the formalism presented in this paper can be used for wider applications as well. If the inhomogeneities are strong enough, Eqs. (7) and (14) can be used for scintillation at directions outside the narrow cones discussed in this paper. This means that, when receivers are arranged in a long chain for the purpose of ordinary ionospheric tomography, the same regions are visible to different receivers at different directions. When a satellite is flying above a chain of receivers distributed along the same magnetic meridian, the integrals in Eq. (14) would be calculated along lines crossing each other in the same regions and they would contain information on the distribution of the variance $\sigma_{N}^{2}$. This means that tomographic methods could be applied in determining the spatial variation of $\sigma_{N}^{2}$.

The principle of amplitude tomography as sketched above actually implies that the density fluctuations remain in the same position and their spatial spectra remain stationary during the interval needed in the tomographic measurement. These conditions are not necessarily fulfilled and therefore the results must be investigated with a critical eye. Even if the spatial variation of $\sigma_{N}^{2}$ were not reliable, however, the method would at least allow finding the regions of irregularities. Then, by means of combining amplitude tomography to the conventional phase tomography, one could investigate the conditions of background ionisation responsible for the instability processes generating the smallscale irregularities.

Acknowledgements. The authors are especially grateful to $\mathrm{O}$. V. Evstafiev for participating the tomographic experiment as well as for his data analysis programme and software development. Thanks are also due to S. M. Chernyakov, J. Pirttilä, P. Pollari, E. Saviaro and T. Ulich for assistance in the tomographic measurements as well as to T. L. Hansen for his great help in arranging the receiver site at Tromsø. This work was supported by the Russian Foundation of Fundamental Research (grant 96-0564274) and the Academy of Finland.

Topical Editor D. Alcaydé thanks J.D. Sahr and another referee for their help in evaluating this paper.

\section{References}

Aarons, J., Global morphology of ionospheric scintillations, Proc. IEEE, 70, 360-378, 1982.
Abramowitz, M., and I. A. Stegun, Handbook of mathematical functions, Dover Publications, New York, 1970.

Baker, K.B., R.A. Greenwald, and R.T. Tsunoda, Very high latitude $\mathrm{F}$ region irregularities observed by $\mathrm{HF}$ radar backscatter, Geophys. Res. Lett., 10, 904-907, 1983.

Briggs, B.H., and A.D. Maude, Spaced sensor observations of pattern motion, J. Geophys. Res., 83, 5309-5311, 1978.

Chernyakov, S.M., E.D. Tereshchenko, B.E. Brunelli, and T. Nygrén, Comparison of ionospheric total electron content measured using the difference Doppler and incoherent scatter methods, Ann. Geophysicae, 11, 10-16, 1993.

Eglitis, P., I.W. McCrea, T.R. Robinson, T.B. Jones, K. Schlegel, and T. Nygrén, Flow dependence of COSCAT spectral characteristics, J. Atmos. Terr. Phys., 58, 189-203, 1996.

Goodman, J.W., Statistical Optics, J Wiley, New York, 1985.

Gradshteyn, I.S., and I.M. Ryzhik, Table of integrals, series and products, Academic Press, New York, 1965.

Haldoupis, C., E. Nielsen, and K. Schlegel, Dependence of radar auroral scattering cross section on the ambient electron density and the destabilizing electric field, Ann. Geophysicae, 8, 195$211,1990$.

Ishimaru, A., Wave Propagation and Scattering in Random Media, Academic Press, Orlando, 1978.

Keskinen, M.J., and S.L. Ossakow, Theories of high-latitude ionospheric irregularities, Radio. Sci., 18, 1077-1091, 1983.

Khudukon, B.Z., E.D. Tereshchenko, A.V. Galinov, A.A. Popov, and T. Nygrén, Determination of drift velocity and anisotropy of irregularities in the auroral ionosphere using radio source scintillation, J. Atmos. Terr. Phys., 56, 93-102, 1994.

Kunitsyn V.E., and E.D. Tereshchenko, Determination of the turbulent spectrum in the ionosphere by a tomographic method J. Atmos. Terr. Phys., 54, 1275-1282, 1992.

Markkanen, M., M. Lehtinen, T. Nygrén, J. Pirttilä, P. Henelius, E. Vilenius, E.D. Tereshchenko, and B.Z. Khudukon, Bayesian approach to satellite radiotomography with applications in the Scandinavian sector, Ann. Geophysicae, 13, 1277-1287, 1995.

Nielsen, E., and K. Schlegel, A first comparison of STARE and EISCAT electron drift velocity measurements, J. Geophys. Res., 88, 5745-5750, 1983.

Nygrén, T., M. Markkanen, M. Lehtinen, E.D. Tereshchenko, B.Z. Khudukon, O.V. Evstafiev, and P. Pollari, Comparison of F region electron density observations by satellite radio tomography and incoherent scatter methods. Ann. Geophysicae, 14, 1422-1428, 1996.

Rytov, S.M., Yu.A. Kratsov, and V.E. Tatarsky, Introduction to statistical radio-physics. Part II. Random Processes. Nauka, Moscow, 1978 (in Russian).

Tsunoda, R.T., High-Latitude F region Irregularities: A Review and Synthesis, Rev. Geophys., 26, 719-760, 1988.

Villain, J.P., G. Caudal, and C. Hanuise, A SAFARI-EISCAT comparison between the velocity of $\mathrm{F}$ region small-scale irregularities and the ion drift, J. Geophys. Res., 90, 84338443, 1985.

Woodman, R.F., On the true velocity in full correlation analysis, Radio Sci., 30, 1459-1465, 1995.

Yeh, K.C., and Liu, C.H., Radio wave scintillation in the ionosphere, Proc. IEEE, 70, 324-360, 1982. 